\title{
Comparison of visual field progression in new-diagnosed primary open-angle and exfoliation glaucoma patients in Sweden
}

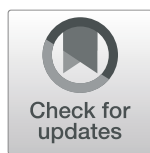

Marcelo Ayala(i)

\begin{abstract}
Background: The present study aimed to compare visual field progression in new-diagnosed exfoliation versus open-angle glaucoma patients.

Methods: Retrospective study. The study included patients with new-diagnosed primary open-angle and exfoliation glaucoma. All patients were followed for 3 years with reliable visual fields. At least five reliable fields were needed for inclusion. Exfoliation and open-angle glaucoma were defined based on the European Glaucoma Society guidelines. Visual field evaluation was performed using the software threshold 24-2 of the Humphrey Field Analysis. Outcomes: Visual field progression. For visual field progression, three different strategies were used: mean deviation (MD), visual field index (VFI), and the guided progression analysis (GPA).

Results: The study included 128 subjects, of the 54 in the open-angle and 74 in the exfoliation glaucoma group. The MD difference values were higher in the exfoliation $(-3.17 \mathrm{~dB})$ than in the primary open-angle $(-1.25 \mathrm{~dB})$ glaucoma group in the three-year follow-up period. The difference between groups was significant (t-test, $p=<$ 0.001). The difference in VFI was calculated for the 3 years follow-up period. The difference was higher in the exfoliation $(-7.65 \%)$ than in the primary open $(-1.90 \%)$ glaucoma group (t-test, $p=<0.001)$. The GPA showed progression in 58\% of cases in exfoliation, and 13\% in primary open glaucoma group (Chi-square, $p=<0.001$ ).

Conclusion: The present study found a more frequent and faster visual field progression in exfoliation than in primary open-angle glaucoma patients. New-diagnosed exfoliation glaucoma patients must be controlled and treated more strictly than primary open-angle glaucoma patients to avoid visual field deterioration.
\end{abstract}

Keywords: Exfoliation glaucoma, Visual field progression, Open-angle glaucoma, Sweden

\section{Background}

Glaucoma is an optic neuropathy that can lead to blindness. It's one of the most common causes of blindness in western countries [1]. Loss of ganglion cells with a consequent loss of visual field characterized the disease. Until now, no treatment has been discovered to cure the disease, and its character is progressive. Still, the cause of glaucoma is unknown, but several risk factors have

Correspondence: marcelo.ayala@ki.se; marcelo.ayala@gu.se Eye Department, Skaraborg Hospital, Skövde, Sahlgrenska Academy, Gothenburg University \& Karolinska Institute, 54185 Skövde, Sweden been described. Increased intraocular pressure (IOP) is the most common risk factor [2]. There are different types of glaucoma; among them, the two most common in Scandinavia are primary open-angle (POAG) and exfoliation glaucoma (EXFG) [3].

Primary open-angle glaucoma (POAG) can be defined as a progressive optic nerve disease that can lead to deterioration of visual field [4]. These changes are usually associated with increased IOP. The term "primary" shows no known cause such as trauma, pigmentation, exfoliation, or inflammation for the development of this

C C The Author(s). 2020 Open Access This article is licensed under a Creative Commons Attribution 4.0 International License, which permits use, sharing, adaptation, distribution and reproduction in any medium or format, as long as you give appropriate credit to the original author(s) and the source, provide a link to the Creative Commons licence, and indicate if changes were made. The images or other third party material in this article are included in the article's Creative Commons licence, unless indicated otherwise in a credit line to the material. If material is not included in the article's Creative Commons licence and your intended use is not permitted by statutory regulation or exceeds the permitted use, you will need to obtain permission directly from the copyright holder. To view a copy of this licence, visit http://creativecommons.org/licenses/by/4.0/ The Creative Commons Public Domain Dedication waiver (http://creativecommons.org/publicdomain/zero/1.0/) applies to the data made available in this article, unless otherwise stated in a credit line to the data. 
glaucoma. Risk factors commonly associated with POAG are age, elevated IOP at baseline, lower central corneal thickness, and family history [5-8].

Exfoliation glaucoma is characterized by the presence of a greyish material that deposits in the anterior chamber of the eye. This material is composed of proteins; the origin of the material is unknown. Exfoliation material has been isolated in different parts of the eye. The pupil, the anterior part of the iris, trabecular meshwork, anterior capsule of the lens, and even in other ocular and extraocular tissues [9-12] showed the presence of exfoliation material. The deposits at the trabecular meshwork occlude the pores at the trabecular meshwork diminishing the outflow, thus increasing the IOP.

As was described above, glaucoma is a progressive disease-glaucoma progression estimates by visual field deterioration. According to previous studies [13, 14], progress shows a large variability among individuals. It's essential to determine the rate of progression in each individual to choose the best treatment modality available; treatment must be individualized. The European Glaucoma Guidelines [4] recommends 5-6 visual fields in 2 years after diagnosing glaucoma to estimate progression. The Swedish Glaucoma Guidelines [15] recommend 5-6 visual fields in 3 years. The approach was judged to be more realistic to the Swedish resources allocation. Still "golden standard" in Sweden for glaucoma progression analysis is visual field testing.

The present study aimed to compare visual field progression in new-diagnosed exfoliation versus open-angle glaucoma patients.

\section{Methods}

The present study was a cohort retrospective chartreview, including all patients with new glaucoma diagnose attending to our Department from 1st January 2012 till 31st December 2015 (4 years). The Ophthalmology Department at the Skaraborg's Hospital is a tertiary unit taking care to around 250,000 inhabitants. The search was performed in our database based on the international classification of diseases (ICD) codes for glaucoma (ICD-10: H409, H401A, and H401C) combined with a code for the first visit (F).

Exclusion criteria:

1) Wrong diagnosis or insufficient information.

2) Patients that cannot perform reliable visual fields at the beginning and/or at least five reliable visual fields 3 years after diagnosis. Reliable visual fields were defined as: false positives $\leq 15 \%$ and/or false negatives $\leq 20 \%$ and/or fixation losses $\leq 30 \%$.

3) Patients with advanced glaucoma damage defined as mean deviation (MD) $\geq 20 \mathrm{~dB}$ and/or visual field index (VFI) $\leq 40 \%$. This criterium was chosen to avoid "floor effects" in which further loss of visual field defects can no longer be detected [16].

4) Patients that required glaucoma surgery during the follow-up period. Uneventful cataract surgery, as well as selective laser treatment (SLT), were not considered as exclusion criteria.

5) Patients were suffering from another eye disease that could alter visual fields under study period like central vein occlusion, retinal detachment, etc.

A detailed medical and ocular history was recorded for each patient. Included patients were referred to the Ophthalmology Department due to high IOP $(\geq 21$ $\mathrm{mmHg}$ ) detected by an optician. Visual acuity, intraocular pressure (IOP) measurements, gonioscopy, optic nerve status, visual field examinations, name and amount of medications, and the presence or absence of exfoliation were recorded. A Snellen chart was used to measure visual acuity. The IOP was measured by Goldmann tonometry. To assess trabecular meshwork, gonioscopy was performed in a dark room using a goniolens with undilated pupils. The presence of Sampaolesi's line was also recorded at the inferior angle. The patient's pupils were dilated with $2.5 \%$ Phenylephrine and Tropicamide 0.5\% (Bausch \& Lomb UK Ltd., 106 London RoadKingston-upon-Thames-Surrey-KT2 6TN-England). Eyes were classified as no exfoliation if there was no evidence of exfoliation material on the pupil, lens, or the angle with dilated pupils. The optic nerve status was studied using a 90-D lens, and the average of vertical cupping was recorded as the cup-to-disc ratio. All patients performed repeated Humphrey Field Analysis (Carl Zeiss, Carl-Zeiss-Straße 22, 73,447 Oberkochen, Germany) using the software threshold 24-2. Only reliable visual fields were considered (see above). Eye-drops were measured as the number of medicines (compounds) and not as the number of bottles. If both eyes were suffering glaucoma, one eye was chosen at random.

For visual field progression analysis, three different methods were used. The first method was based on the general parameter called "mean deviation" (MD). This parameter was chosen since several studies still use MD as an indicator of progression $[14,17,18]$. Cataract development can also alter MD. The second method used was the progression analysis based on the "visual field index" (VFI). The device calculated the VFI automatically. The device also performed a regression analysis and expressed if the regression curve (slope) statistically significant deviates from the expected values. The "rate of progression" is calculated as the amount of VFI deterioration (\%)/year. This kind of progression analysis is also called as "trend analysis." The third progression analysis studied was the guided progression analysis (GPA). Also included in the device and performed automatically. The 
GPA is, as difference with the previous, "an event analysis." The machine compares every single point to prior examinations. A progression analysis is performed (GPA); the results can be: no, possible or likely progression. For analysis purposes in this study, the results were evaluated as "no progression" or "progression," which included both "possible" and "likely" progression.

Exfoliation glaucoma was defined as untreated IOP of $21 \mathrm{mmHg}$ or beyond, open anterior chamber angle in gonioscopy, glaucomatous visual field defect (at least two repeatable Humphrey 24-2) and glaucomatous optic nerve damage, concomitant with the presence of exfoliation material, observed at the anterior lens capsule and/ or at the pupillary border according to the definition of the European Glaucoma Society [4]. Primary open-angle glaucoma (POAG) was defined in the same manner but without the presence of exfoliation material in a dilated pupil.

The study adhered to the tenets of the declaration of Helsinki Declaration.

\section{Statistics}

For statistical analysis, SPSS (IBM, 1 New Orchard Road Armonk, NY 10504, USA) software was used. The continuous variables (IOP, MD, and VFI) were tested for normality using the Kolmogorov-Smirnov test and for homoscedasticity using the Levene's test. The continuous variables were tested for significance using the t-test, and the Chi-square test was used for testing noncontinuous variables (GPA). The significance level was set at 0.05 .

\section{Results}

Totally, 168 patients suffering from newly diagnosed glaucoma were identified in the 4 years recruiting period. Of them, 40 patients were excluded and the rest, 128 patients were included and analyzed.

Excluded cases are shown in Table 1. A greater number of cases were excluded in the exfoliation $(n=25)$ vs. the primary open-angle glaucoma group $(n=15)$. The most common reason for exclusion in both groups was advanced visual field damage at the beginning. This reason accounted for $56 \%$ in the exfoliation and $40 \%$ in the primary open-angle glaucoma group. Trabeculectomy was the second reason for exclusion. It accounted for $36 \%$ in exfoliation and $20 \%$ in primary open-angle glaucoma patients.

Included patients were 128 subjects, of the 54 in the primary open-angle, and 74 in the exfoliation group. The baseline parameters of the included patients are shown in Table 2. It's interesting to observe that visual acuity was lower in the exfoliation than the primary open-angle glaucoma group though age was not significantly different. Also, exfoliation glaucoma presented more often unilateral than bilateral (78 vs. $22 \%)$.

Another parameter that differed considerably among the groups was the IOP at the beginning of the study. The average IOP in exfoliation was $32.9 \mathrm{mmHg}$; meanwhile, it was $27.6 \mathrm{mmHg}$ in the primary open-angle glaucoma patients (t-test, $p<0.001)$.

The mean deviation (MD), pattern standard deviation (PSD), and visual field index (VFI) did not differ among the groups at the beginning of the study. No difference in the studied parameters can be explained by the fact that many patients with advanced visual field defects were excluded in the exfoliation than in the primary open-angle glaucoma group.

The continuous variables: IOP, MD, and VFI were tested for normality and homoscedasticity. All variables were normally distributed according to the KolmogorovSmirnov test (IOP: $p=0.127$; MD: $p=0.244$; VFI, $p=$ 0.182). Regarding homoscedasticity, the Levene's test also showed equality of variances in all the variables (IOP, $p=0.623$; MD, $p=0.092$; VFI, $p=0.067$ ).

The results of the study after 3 years follow-up are shown in Table 3. The difference in the mean deviation (MD) between the beginning and end of the study (3 years follow-up) was calculated for both groups. The MD difference values were higher in the exfoliation $(3.17 \mathrm{~dB})$ than in the primary open $(1.25 \mathrm{~dB})$ glaucoma group. The difference between groups was significant ( $\mathrm{t}$ test, $p=<0.001$ ).

The difference in VFI was calculated for the 3 years follow-up period. The difference was higher in the exfoliation $(7.65 \%)$ than in the primary open $(1.90 \%)$ glaucoma group. The difference was statistically significant (t-test, $p=<0.001)$. The device also automatically gave a calculation of VFI deterioration/year (Rate of

Table 1 Exclusion criteria

\begin{tabular}{lll}
\hline Cause & POAG & EXFG \\
\hline Advanced Visual Field damage (MD $\geq 20 \mathrm{~dB}$ and/or VFI $\leq 40 \%)$ & $6(40 \%)$ & $14(56 \%)$ \\
Trabeculectomy & $3(20 \%)$ & $9(36 \%)$ \\
No reliable visual fields & $3(20 \%)$ & $1(4 \%)$ \\
Other diseases & $3(20 \%)$ & $1(4 \%)$ \\
Total & $15(100 \%)$ & $25(100 \%)$ \\
\hline
\end{tabular}

POAG primary open-angle glaucoma, EXFG exfoliation glaucoma, MD mean deviation, VFI visual field index 
Table 2 Baseline demographic and clinical characteristics on the patients included in the study $(n=168)$

\begin{tabular}{|c|c|c|c|c|}
\hline & POAG & EXFG & Test & $P$ \\
\hline Age (years) (SD) & $69.6(8.69)$ & $71.4(6.39)$ & T-test & 0.20 \\
\hline Gender (M/W) (\%) & $28 / 26(52 / 48)$ & 29/35 (45/55) & Chi-square & 0.57 \\
\hline Visual acuity (Snellen) (SD) & $0.91(0.11)$ & $0.84(0.21)$ & T-test & $0.027^{*}$ \\
\hline Phakia/pseudophakia (\%) & $50 / 4(92.5 / 7.5 \%)$ & 60/4 (93.75/6.25\%) & Chi-square & 0.34 \\
\hline Bilateral/unilateral (\%) & $37 / 17(69 / 31 \%)$ & $14 / 50(22 / 78 \%)$ & Chi-square & $<0.001^{*}$ \\
\hline Intraocular pressure (IOP) (mmHg) (SD) & $27.62(5.4)$ & $32.9(4.81)$ & T-test & $<0.001^{*}$ \\
\hline Mean deviation (MD) (dB) (SD) & $-5.57(4.83)$ & $-5.65(4.52)$ & T-test & 0.92 \\
\hline Pattern deviation (PSD) (dB) (SD) & $5.10(3.81)$ & $4.41(3.14)$ & T-test & 0.28 \\
\hline Visual field index (VFI) (\%) (SD) & $87.49(12.45)$ & $87.65(12.65)$ & T-test & 0.94 \\
\hline
\end{tabular}

POAG primary open-angle glaucoma, EXFG exfoliation glaucoma

$\left(^{*}\right)=$ Significant values

progression/year). The decrease of VFI/year was $2.17 \%$ for exfoliation and $0.54 \%$ for the primary open glaucoma group. The difference was also statistically significant (t-test, $p=<0.001$ ).

Regarding the proportion of cases that showed progression or not in the trend-based (VFI) showed significant progress in $50 \%$ of exfoliation and $15 \%$ of primary open glaucoma. The difference was significant (Chisquare, $p=<0.001)$. Similar results were found when checking for the event analysis strategy, guided progression analysis (GPA). Using the GPA, 58\% showed progression in the exfoliation, and $13 \%$ in the primary open glaucoma group. The difference was significant (Chisquare, $p=<0.001)$.

The average number of medications used at the end of the study was higher in the exfoliation (2.80) than in the primary open (1.75) glaucoma group. The difference was statistically significant (t-test, $p=<0.001)$. The number of SLT treatments performed was also higher in the exfoliation (19\%) than in the primary open (4\%) glaucoma group. The difference was significant (Chi-square, $p=<0.005$ ).

\section{Discussion}

The present study showed a more frequent and faster visual field deterioration among exfoliation than primary open-angle glaucoma patients. The results are probably not surprising; what is new in this study is that all included patients were new-diagnosed as glaucoma. Previous studies [2, 19] measuring visual field progression included both new and old diagnosed patients. The other interesting point to raise is that very few published studies have included exfoliation patients. The significant majority of studies measuring progression included only open-angle glaucoma patients $[14,20,21]$.

The results coming from this study are in accord with results coming from the Early Manifest Glaucoma Trial (EMGT) [19] that showed an increased visual field progression in exfoliation than in primary open glaucoma patients. The authors found a progression of MD: -3.13 $\mathrm{dB} /$ year, a higher value than the one found in this study $(-1.05 \mathrm{~dB} /$ year). The higher values found in the EMGT can be attributed to the fact that patients were untreated. In the EMGT, the VFI values were not studied; since VFI was not available at the time. Kim et al. [22]

Table 3 Results after three years follow-up

\begin{tabular}{|c|c|c|c|c|}
\hline & POAG & EXFG & Test & $P$-value \\
\hline Average difference $M D$ in 3 years (dB) (SD) & $1.25(1.63)$ & $3.17(2.26)$ & T-test & $P=<0.001^{*}$ \\
\hline Average difference in VFI in 3 years (\%) (SD) & $1.90(1.45)$ & $7.66(5.68)$ & T-test & $P=<0.001^{*}$ \\
\hline Average rate of progression VFI (\%/year) (SD) & $-0.54(1.07)$ & $-2.17(2.08)$ & T-test & $P=<0.001^{*}$ \\
\hline Progress/No progress VFI (\%) & $8 / 46(15 / 85)$ & $32 / 32(50 / 50)$ & Chi-square & $P=<0.001^{*}$ \\
\hline Progress/No progress GPA (\%) & $7 / 47(13 / 87)$ & $37 / 27(58 / 42)$ & Chi-square & $P=<0.001^{*}$ \\
\hline Average IOP $(\mathrm{mmHg})(\mathrm{SD})$ at the end of the study & $15.93(2.56)$ & $16.34(2.68)$ & T-test & $P=0.40$ \\
\hline Average number of medications (SD) & $1.75(0.84)$ & $2.80(0.80)$ & T-test & $P=<0.001^{*}$ \\
\hline Number of SLT/ No SLT (\%) & $2 / 52(4 / 96)$ & 10/54 (19/81) & Chi-square & $P=0.002^{*}$ \\
\hline Number of Cataract operation/ No cataract operation (\%) & $4 / 50(7 / 93)$ & $11 / 53(17 / 83)$ & Chi-square & $P=0.02^{*}$ \\
\hline
\end{tabular}

POAG primary open-angle glaucoma, EXFG exfoliation glaucoma, MD mean deviation, SD standard deviation, VFI visual field index, GPA guided progression analysis, IOP intraocular pressure, SLT selective laser trabeculoplasty

$(*)=$ Significant values 
published a study identifying risk factors for visual field progression in a retrospective cohort study, including 1317 eyes. They found exfoliation as a risk factor for progression. Visual field progression was MD: $-0.14 \mathrm{~dB} /$ year in the POAG and $-0.24 \mathrm{~dB} /$ year in the exfoliation group. Regarding VFI, they described a deterioration of $-0.32 \% /$ year in the POAG and $-0.59 \%$ /year in the exfoliation group. The present study found a progression in the VFI values of $-0.54 \%$ /year in POAG and $-2.17 \%$ / year for exfoliation.

Interestingly, Kim et al. study included only $4.4 \%$ of patients suffering from exfoliation glaucoma. Relative similar results in visual field progression were reported by Kocaturk et al. [23] The authors reported a VFI deterioration of $-0.3 \% /$ year in the POAG $(n=146)$ and $0.43 \% /$ year in the exfoliation $(n=123)$ group.

The study had an extended follow-up of 15 years. All the previously mentioned studies showed a difference in progression between the different types of glaucoma. However, Moraes et al. [24] showed different results, calculating exfoliation as a risk factor for progress in a multivariate analysis. Among the included glaucomas, exfoliation glaucoma patients had higher rates of visual field progression than POAG, normal-tension glaucoma (NTG), etc. The authors found a visual field progression of $-0.65 \mathrm{~dB} /$ year in MD values. However, the increased risk associated with exfoliation became nonsignificant after adjusting for baseline and intercurrent variables, suggesting the influence of other ocular parameters, such as higher IOP peaks, more common in exfoliation glaucoma patients. These results must be confirmed in further studies. Comparisons among studies are difficult to perform due to differences in follow-up extension, exclusion/inclusion criteria, ethnicity, age, treatments, etc.

The present study showed a faster visual field progression in MD values compared to previous studies. It's possible to speculate that the difference can be attributed to the fact that all included patients were newdiagnosed glaucoma subjects. After establishing diagnose, the patients were treated, and the visual field deterioration diminished compared with the high progression values the disease has in its natural history (without treatment) [19]. It's possible to speculate that the disease's progression slows down with the right treatment after some years. Another possible explanation to the results found in this study can be genetic; all included patients were of Scandinavian origin. Previous studies [25] showed differences in gene expression among different populations studied.

All included patients were sent to the Ophthalmology Department at the Skaraborg's Hospital by opticians. Opticians referred the patients when they showed high IOP $(\geq 21 \mathrm{mmHg})$. The way the patients came into the Department created a selection bias, but on the other hand, this is the way we got patients in our Department. Patients suffering from exfoliation but with lower IOP were not included in this study because the optometrists did not send them. Exfoliation glaucoma usually presents as a high IOP glaucoma, so the number of patients with normal IOP suffering from exfoliation glaucoma might be quite low.

Though a good IOP reduction due to medication and laser, exfoliation glaucoma patients continued to progress. The average IOP at the end of the study in exfoliation patients was $16.34 \mathrm{mmHg}$. It seems that even IOP was quite low, the IOP reduction was not enough to stop the visual field deterioration, and bigger efforts might be necessary to slow down the disease.

All patients got an estimated "target pressure" after their glaucoma diagnose. This target pressure is a clinical-based IOP level based on the patient's age, bilateral or unilateral presentation of glaucoma, visual field damage, etc. In our clinical practice, a normal target pressure is an IOP around $20 \mathrm{mmHg}$ or below when establishing glaucoma. The IOP "target pressure" always re-evaluates after 3 years when we have the data coming from the visual field examinations. Based on this study's results, it seems that exfoliation glaucoma patients must be considered to need a lower target pressure than POAG patients.

At the beginning of the study, visual fields were similar in both groups though a higher IOP was measured in the exfoliation group. This point must be interpreted with caution. One can think that exfoliation glaucoma with elevated IOP at diagnose has similar visual field deterioration than POAG patients with lower IOP. Similar visual field deterioration at the beginning of the study explains by the fact that advanced visual field defects were excluded to avoid "ceiling effects." Exclusion due to very advanced visual field defects was more common in the exfoliation than in the POAG group. Exfoliation glaucoma patients showed not only a faster visual field deterioration but also a larger inter-individual variability. Some patients showed nearly no progress; meanwhile, others progressed very fast. Genetic differences can probably explain this difference in disease progression [25].

This study has some limitations. One is its retrospective design. Though medical records were standardized for glaucoma patients, it's possible for some missing data. Reporting bias is also a common problem in retrospective studies. One of the most significant limitations of the study is the exclusion of very advanced glaucoma subjects. Patients were excluded to avoid "ceiling effects." Unfortunately, there is still no suitable method to evaluate glaucoma progression in advanced cases. Another limitation is that the study did not include data about anatomical progress, like optical coherence 
tomography (OCT) or photographs. At the time the study was performed, it was not a common clinical practice to study our glaucoma patients with OCT. Still, there is no consensus about which method is the best to evaluate glaucoma progression [26].

The present study showed a higher amount of unilateral glaucoma patients in the exfoliation (78\%) than in the open-angle (31\%) glaucoma group. Higher unilaterality is in accord with previously published studies [3, 27]. All patients were examined with dilated pupils, so the risk to miss the presence of exfoliation was quite low. The presence of exfoliation correlated well with high IOP in the affected eyes. Sometimes the difference between eyes was around $20-30 \mathrm{mmHg}$. No patient "converted" from unilateral to bilateral during the 3 years follow-up period. According to previous studies [28], approximately $38 \%$ of patients converted to bilateral in a 10-year follow-up period. The reason for the unilateral expression of the disease is still unknown. Possibly the condition is bilateral but with an asymmetrical phenotypical expression [29]. Genetic studies have been performed showing that possibly local factors at the eye determine the expression or not of the genotype [30].

\section{Conclusions}

In conclusion, using three different strategies for testing visual field progression it was found a more frequent and faster visual field progression in exfoliation than in primary open-angle glaucoma patients. Exfoliation glaucoma patients must be controlled and treated more strictly than primary open-angle glaucoma patients to avoid visual field deterioration.

\section{Abbreviations}

POAG: Primary open-angle glaucoma; EXFG: Exfoliation glaucoma; MD: Mean deviation; GPA: Guided progression analysis; VFI: Visual field index; IOP: Intraocular pressure; ICD: International classification of diseases; EMGT: Early manifest glaucoma trial; OCT: Optical coherence tomography

\section{Acknowledgements}

Not applicable.

\section{Author's contributions}

There is only one author in this study (MA). The author read and approved the final manuscript.

\section{Funding}

Open access funding provided by Karolinska Institute.

\section{Availability of data and materials}

The datasets used and/or analyzed during the current study are available from the corresponding author on reasonable request.

\section{Ethics approval and consent to participate}

The study was approved by the Swedish Ethical Authority, Uppsala, DN 2020-03175. Written consent from the patients was not necessary for this non-interventional retrospective chart-review study. No administrative permissions were required to access the raw data from the database. Patient data were de-identified prior to data collection.
Consent for publication

Not applicable.

\section{Competing interests}

The author declares no competing interests.

Received: 11 June 2020 Accepted: 29 July 2020

Published online: 05 August 2020

\section{References}

1. Resnikoff S, Pascolini D, Etya'ale D, Kocur I, Pararajasegaram R, Pokharel GP, et al. Global data on visual impairment in the year 2002. Bull WHO. 2004; 82(11):844-51

2. Leske MC, Heijl A, Hussein M, Bengtsson B, Hyman L, Komaroff E. Factors for glaucoma progression and the effect of treatment: the early manifest glaucoma trial. Arch Ophthalmol. 2003;121(1):48-56.

3. Aström $S$, Stenlund $H$, Lindén $C$. Incidence and prevalence of pseudoexfoliations and open-angle glaucoma in northern Sweden: II. Results after 21 years of follow-up. Acta Ophthalmol Scand. 2007;85(8):832-7.

4. European Glaucoma Society Terminology and Guidelines for Glaucoma. Chapter 2: classification and terminologySupported by the EGS Foundation: part 1: foreword; introduction; glossary; chapter 2 classification and terminology. Br J Ophthalmol. 2017;101(5):73-127.

5. Zhou K, Shang X, Wang XY, Wang XJ, Cheng HH, Hu HS, et al. Risk factors for visual field loss progression in patients with primary open-angle glaucoma in Wenzhou area. Zhonghua Yan Ke Za Zhi. 2019;55(10):777-84.

6. Mowatt L. Risk factors for rapid Glaucoma disease progression. Am J Ophthalmol. 2018;186:170-1.

7. Chan TCW, Bala C, Siu A, Wan F, White A. Risk factors for rapid Glaucoma disease progression. Am J Ophthalmol. 2017;180:151-7.

8. Actis AG, Versino E, Brogliatti B, Rolle T. Risk factors for primary open angle Glaucoma (POAG) progression: a study ruled in Torino. Open Ophthalmol J. 2016;10:129-39.

9. Ritch R, Schlotzer-Schrehardt U. Exfoliation syndrome. Surv Ophthalmol. 2001;45(4):265-315.

10. Dewundara S, Pasquale LR. Exfoliation syndrome: a disease with an environmental component. Curr Opin Ophthalmol. 2015;26(2):78-81.

11. Ritch R. Ocular and systemic manifestations of exfoliation syndrome. J Glaucoma. 2014;23(8 Suppl 1):S1-8.

12. Pasquale $L R$, Borras $T$, Fingert $J H$, Wiggs $J$, Ritch R. Exfoliation syndrome: assembling the puzzle pieces. Acta Ophthalmol. 2016;94(6):e505-12

13. Baek SU, Kim YK, Park KH, Jeoung JW. Long-term follow-up on Glaucoma patients with initial single-Hemifield defect: progression patterns and associated factors. J Glaucoma. 2019;28(12):1041-7.

14. Salonikiou A, Founti $P$, Kilintzis V, Antoniadis A, Anastasopoulos E, Pappas T, et al. Tolerable rates of visual field progression in a population-based sample of patients with glaucoma. Br J Ophthalmol. 2018;102(7):916-21.

15. Heijl A, Alm A, Bengtsson B, Bergstrom A, Calissendorff B, Lindblom B, et al. The Glaucoma Guidelines of the Swedish Ophthalmological Society. Acta Ophthalmol Suppl. 2012;251:1-40

16. Wall M, Woodward KR, Doyle CK, Artes PH. Repeatability of automated perimetry: a comparison between standard automated perimetry with stimulus size III and V, matrix, and motion perimetry. Invest Ophthalmol Vis Sci. 2009:50(2):974-9.

17. Liebmann K, De Moraes CG, Liebmann JM. Measuring rates of visual field progression in linear versus nonlinear scales: implications for understanding the relationship between baseline damage and target rates of Glaucoma progression. J Glaucoma. 2017:26(8):721-5.

18. Berchuck SI, Mukherjee S, Medeiros FA. Estimating rates of progression and predicting future visual fields in Glaucoma using a deep Variational autoencoder. Sci Rep. 2019;9(1):18113.

19. Heijl A, Bengtsson B, Hyman L, Leske MC. Natural history of open-angle glaucoma. Ophthalmology. 2009;116(12):2271-6.

20. Zhang X, Parrish RK 2nd, Greenfield DS, Francis BA, Varma R, Schuman JS, et al. Predictive factors for the rate of visual field progression in the advanced imaging for Glaucoma study. Am J Ophthalmol. 2019;202:62-71.

21. Nguyen AT, Greenfield DS, Bhakta AS, Lee J, Feuer WJ. Detecting Glaucoma progression using guided progression analysis with OCT and visual field assessment in eyes classified by international classification of disease severity codes. Ophthalmol Glaucoma. 2019;2(1):36-46. 
22. Kim JH, Rabiolo A, Morales E, Yu F, Afifi AA, Nouri-Mahdavi K, et al. Risk factors for fast visual field progression in Glaucoma. Am J Ophthalmol. 2019; 207:268-78.

23. Kocaturk T, Bekmez S, Katranci M, Cakmak H, Dayanir V. Long term results of visual field progression analysis in open angle Glaucoma patients under treatment. Open Ophthalmol J. 2015;9:116-20.

24. De Moraes CG, Liebmann JM, Liebmann CA, Susanna R Jr, Tello C, Ritch R. Visual field progression outcomes in glaucoma subtypes. Acta Ophthalmol. 2013;91(3):288-93.

25. Founti $P$, Haidich $A B$, Chatzikyriakidou A, Salonikiou A, Anastasopoulos $E$ Pappas T, et al. Ethnicity-based differences in the association of LOXL1 polymorphisms with Pseudoexfoliation/Pseudoexfoliative Glaucoma: a meta-analysis. Ann Hum Genet. 2015:79(6):431-50.

26. Vianna JR, Chauhan BC. How to detect progression in glaucoma. Prog Brain Res. 2015;221:135-58.

27. Musch DC, Shimizu T, Niziol LM, Gillespie BW, Cashwell LF, Lichter PR. Clinical characteristics of newly diagnosed primary, pigmentary and pseudoexfoliative open-angle glaucoma in the collaborative initial Glaucoma treatment study. Br J Ophthalmol. 2012;96(9):1180-4

28. Puska PM. Unilateral exfoliation syndrome: conversion to bilateral exfoliation and to glaucoma: a prospective 10-year follow-up study. J Glaucoma. 2002; 11(6):517-24.

29. Hammer T, Schlotzer-Schrehardt U, Naumann GO. Unilateral or asymmetric pseudoexfoliation syndrome? An ultrastructural study. Arch Ophthalmol. 2001;119(7):1023-31

30. Ayala M, Cuklev F. Indistinguishable gene expression between healthy eyes and eyes with unilateral exfoliative glaucoma. Clin Ophthalmol. 2018;12: $1155-62$.

\section{Publisher's Note}

Springer Nature remains neutral with regard to jurisdictional claims in published maps and institutional affiliations.

Ready to submit your research? Choose BMC and benefit from:

- fast, convenient online submission

- thorough peer review by experienced researchers in your field

- rapid publication on acceptance

- support for research data, including large and complex data types

- gold Open Access which fosters wider collaboration and increased citations

- maximum visibility for your research: over $100 \mathrm{M}$ website views per year

At BMC, research is always in progress.

Learn more biomedcentral.com/submissions 